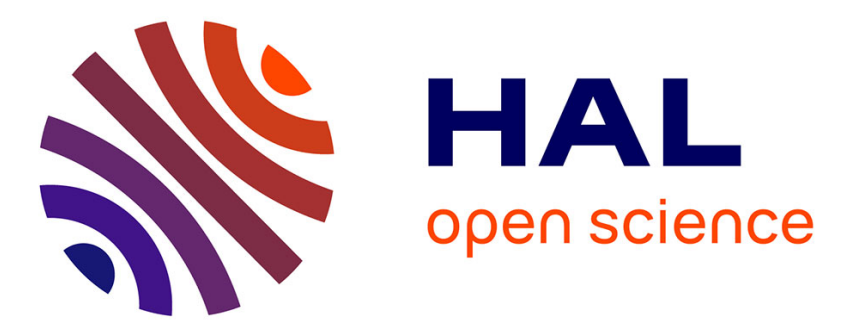

\title{
The Cross-Polar Cap Saturation in GUMICS-4 During High Solar Wind Driving
}

\author{
A. Lakka, I. Pulkkinen, A. Dimmock, M. Myllys, I. Honkonen, M. Palmroth
}

\section{To cite this version:}

A. Lakka, I. Pulkkinen, A. Dimmock, M. Myllys, I. Honkonen, et al.. The Cross-Polar Cap Saturation in GUMICS-4 During High Solar Wind Driving. Journal of Geophysical Research Space Physics, 2018, 123 (5), pp.3320-3332. 10.1002/2017JA025054 . insu-01744909

\section{HAL Id: insu-01744909 \\ https://hal-insu.archives-ouvertes.fr/insu-01744909}

Submitted on 24 Oct 2018

HAL is a multi-disciplinary open access archive for the deposit and dissemination of scientific research documents, whether they are published or not. The documents may come from teaching and research institutions in France or abroad, or from public or private research centers.
L'archive ouverte pluridisciplinaire HAL, est destinée au dépôt et à la diffusion de documents scientifiques de niveau recherche, publiés ou non, émanant des établissements d'enseignement et de recherche français ou étrangers, des laboratoires publics ou privés. 


\section{Journal of Geophysical Research: Space Physics}

\section{RESEARCH ARTICLE}

10.1002/2017JA025054

Key Points:

- The ionospheric cross-polar cap potential (CPCP) response to the solar wind electric field $Y$ component in GUMICS-4 is similar to other codes - The nature of the saturation of the CPCP in GUMICS-4 is heavily affected by magnitude of the solar wind Alfvén Mach number $M_{A}$

- Low upstream $M_{A}$ during simulation initialization can cause differences in the cross-polar cap potential value of order several kV

Correspondence to:

A. Lakka,

antti.lakka@aalto.fi

Citation:

Lakka, A., Pulkkinen, T. I., Dimmock, A. P., Myllys, M., Honkonen, I., \& Palmroth, M. (2018). The cross-polar cap saturation in GUMICS-4 during high solar wind driving. Journal of Geophysical Research: Space Physics, 123, 3320-3332. https://doi.org/10.1002/2017JA025054

Received 29 NOV 2017 Accepted 22 MAR 2018 Accepted article online 26 MAR 2018 Published online 2 MAY 2018

(C)2018. American Geophysical Union. All Rights Reserved.

\section{The Cross-Polar Cap Saturation in GUMICS-4 During High Solar Wind Driving}

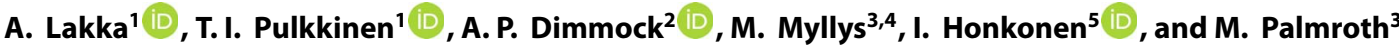 \\ ${ }^{1}$ Department of Electronics and Nanoengineering, Aalto University, Espoo, Finland, ${ }^{2}$ Swedish Institute of Space Physics, \\ Uppsala, Sweden, ${ }^{3}$ Department of Physics, University of Helsinki, Helsinki, Finland, ${ }^{4}$ Laboratoire de Physique et Chimie de \\ I'Environnement et de l'Espace, CNRS, Orléans, France, ${ }^{5}$ Finnish Meteorological Institute, Helsinki, Finland
}

\begin{abstract}
It is well known that the Earth's ionospheric cross-polar cap potential (CPCP) saturates as a response to the solar wind (SW) driver especially when the level of driving is high and the interplanetary magnetic field is oriented southward. Moreover, previous studies have shown that the upstream Alfvén Mach number may be an important factor in the saturation effect. While the CPCP is often viewed as a measure of the SW-magnetosphere-ionosphere coupling, the processes associated with the nonlinearity of the coupling remain an open issue. We use fourth edition of the Grand Unified Magnetosphere-lonosphere Coupling Simulation (GUMICS-4) and artificial SW data to mimic weak and strong driving in order to study the CPCP response to a wide range of interplanetary magnetic field magnitudes $(3.5-30 \mathrm{nT})$ and upstream Alfvén Mach number values (1.2-22). The results provide the first overview of the CPCP saturation in GUMICS-4 and show that the onset of saturation is strongly dependent on the upstream Alfvén Mach number and the physical processes responsible for the saturation effect might take place both in the Earth's magnetosheath and in the upstream SW.
\end{abstract}

\section{Introduction}

It is well known that the solar wind (SW) and the Earth interact more efficiently when the interplanetary magnetic field (IMF) is oriented southward (IMF $B_{Z}<0 \mathrm{nT}$; Koustov et al., 2009; Nishida, 1968). This has led to a conclusion that magnetic reconnection is mostly responsible for transporting mass, momentum, and energy from the SW to the magnetosphere (Dungey, 1961). During the convection cycle the dayside magnetospheric field and the IMF merge and as a consequence magnetic flux is transported over the polar cap ionosphere and plasma to the nightside where it may reconnect again. The magnetic flux transport is a driver of a two-cell plasma convection pattern in the ionosphere (Hill, 1994), where antisunward flow takes place across the polar cap and sunward return flow at lower latitudes. The rate at which magnetic flux crosses the merging line is equal to electric potential drop along that line according to Faraday's law of induction. This potential drop is transmitted to the polar ionosphere along the field lines and measured as the cross-polar cap potential (CPCP; Crooker, 1988; Lockwood, 1991; Lockwood \& Smith, 1992; Siscoe \& Huang, 1985). There are also other factors contributing to the CPCP such as viscous interaction owing to interaction between the magnetosheath flows and the flanks of the magnetosphere as was first proposed by Axford and Hines (1961) and the nightside reconnection component (Milan, 2004) that is, unlike its dayside counterpart, not directly controlled by the SW such that its contribution to the CPCP prevails even during the absence of dayside reconnection. The existence of these other factors is supported by the fact that even if the IMF $B_{Z}>0 \mathrm{nT}$, some measurable residual potential still exists. However, several studies have shown that other factors make up perhaps only $10 \%$ of the total ionospheric potential (see, e.g., Newell et al., 2008; Reiff et al., 1981) and it can thus be said that the dayside reconnection is the major contributor to the CPCP. Therefore, the CPCP can be viewed as a measure of the coupling between the ionosphere, the magnetosphere, and the SW.

It was long assumed that the dependence of the CPCP on the upstream conditions is linear (Reiff, 1986; Reiff et al., 1981). Saturation occurs during high driving conditions (see, e.g., Russell et al., 2001), which are statistically rare: when the SW speed is high $(V>400 \mathrm{~km} / \mathrm{s})$, the IMF magnitude is mostly in the range of 2-8 nT (Dimmock et al., 2014), while during interplanetary coronal mass ejections (ICMEs), the field magnitude can easily go above $10 \mathrm{nT}$ (Myllys et al., 2016). ICME events typically drive the strongest geomagnetic disturbances and thus generate conditions that lead to CPCP saturation. Russell et al. (2001) studied five geomagnetic 
storms and found that the saturation IMF magnitude was around $7 \mathrm{nT}$. Assuming purely southward IMF and median SW velocity $\left(440 \mathrm{~km} / \mathrm{s}\right.$ ), reconnection electric field given by $V B_{Z}$ is $3 \mathrm{mV} / \mathrm{m}$ for the saturation. As presented by Shepherd (2007) limit values for the saturation electric field vary significantly depending on the study; we conclude that the saturation effect takes place somewhere between $E_{\mathrm{SW}}=0.5$ and $10 \mathrm{mV} / \mathrm{m}$ with saturation potential residing below $300 \mathrm{kV}$.

Many models have been proposed to explain the physics behind the saturation process. Previously, these models were divided into two groups in the literature: reconnection models and postreconnection models depending on whether the processes related to the CPCP saturation take place before or after the SW plasma reconnects with the geomagnetic field. The models include the Siscoe-Hill model (Hill et al., 1976; Siscoe et al., 2002), which argues that the rate of reconnection is lowered because the Alfvén speed is reduced at the reconnection site due to strong dawn-to-dusk cross-polar cap currents reducing the magnetic field strength in the dayside magnetosphere (reconnection model). An example of a postreconnection model is the ionospheric outflow model (Winglee et al., 2002), which argues that an increase of driving of the magnetosphere by the SW causes an increase in the ionospheric-plasma outflow into the magnetosphere and this plasma loads mass to the magnetosphere, which in turn reduces the potential due to slowing down of the flow.

None of these models is well above others when it comes to observational evidence, which may be due to multiple parameters controlling the saturation process (see, e.g., Borovsky et al., 2009). Hence, dividing the models in reconnection and postreconnection models appears not to be the best way to classify them. The effect of ionospheric conductivity on the saturation process has been proposed by multiple authors (see, e.g., Nagatsuma, 2004; Ridley et al., 2004). Recently, Kubota et al. (2017) studied the effect of ionospheric auroral conductivity on the CPCP saturation during a strong coronal mass ejection event that occurred on 15 July 2000. It was found out that the inclusion of auroral conductivity in the ionospheric part of the global magnetohydrodynamic (MHD) model by Tanaka (1994) leads to saturated CPCP without any effect on the field-aligned currents, thus suggesting that a current system is created with a dynamo region in the magnetosphere and a dissipation region in the ionosphere. Moreover, it has been shown by multiple authors (Lopez et al., 2010; Myllys et al., 2016; Ridley, 2005, 2007; Wilder et al., 2015) that the upstream Alfvén Mach number plays a part in the process. Lopez et al. (2010) explained the saturation effect owing to the magnetosheath becoming $\mathbf{J} \times \mathbf{B}$ force dominated instead of being pressure gradient force dominated, when the IMF magnitude is large and the Alfvén Mach number is less than 4. Observational support for the theories that saturation is taking place due to magnetosheath dynamics is shown by a statistical study by Pulkkinen et al. (2016) such that the energy input from the magnetosheath to the magnetosphere is not linearly dependent on the energy incident in the SW upstream of the bow shock, while there is linear correlation between the Poynting flux at the magnetopause and the directly driven auroral electrojets (AEs).

In this paper we examine conditions during which the CPCP saturation occurs in the fourth edition of the Grand Unified Magnetosphere-lonosphere Coupling Simulation (GUMICS-4; Janhunen et al., 2012) global MHD simulation. We also investigate where in the Earth's space environment the processes leading to saturation might take place. We carry out series of simulations with artificial SW input, which covers both high and low SW driving by combining high and low IMF magnitudes with high and low SW plasma flow speeds. We execute runs with different IMF magnitudes (3.5-30 nT) and change the upstream SW speed in a similar way in each run (350-750 km/s). Since the SW density is fixed, the upstream Alfvén Mach number value varies in the range 1.2-22. As a consequence, we create conditions that vary from conditions featuring large Alfvén Mach number to almost sub-Alfvénic conditions.

This paper is ordered in a following manner: section 2 describes GUMICS-4 global MHD code and the simulation runs, section 3 highlights the main results, and the discussion and conclusions can be found at the end of the paper.

\section{Methods}

\subsection{GUMICS-4}

All simulations in this study were run using the GUMICS-4, which couples 3-D MHD magnetosphere with a spherical electrostatic ionosphere (Janhunen et al., 2012). The MHD solver utilizes finite volume method and solves the ideal MHD equations inside a simulation box that has dimensions of $32 \ldots-224 R_{E}$ in $X_{\mathrm{GSE}}$ direction and $-64 \ldots+64 R_{E}$ in both $Y_{\mathrm{GSE}}$ and $Z_{\mathrm{GSE}}$ directions. The inner boundary is spherical with a radius of $3.7 R_{E}$. The magnetosphere is coupled to the ionosphere using dipole mapping of the field-aligned current pattern 


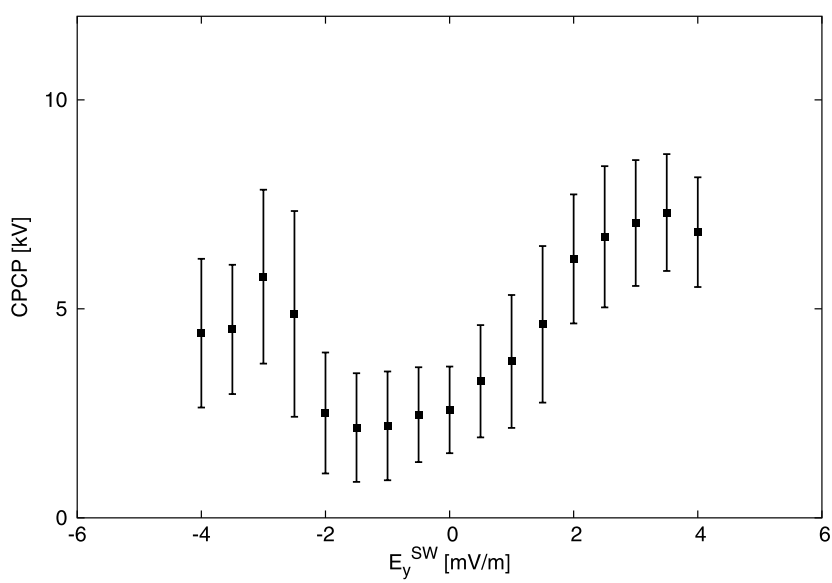

Figure 1. The cross-polar cap potential (CPCP) as a function of the interplanetary magnetic field $E_{Y}$ during 10-day long time period in GUMICS-4 simulation (the details of the period can be found at Lakka et al., 2017). The data are binned by interplanetary magnetic field $E_{Y}$ with $0.5-\mathrm{mV} / \mathrm{m}$ intervals and the CPCP is averaged within these bins. Standard deviations computed for each bin are used as error bars. and the electron precipitation from the magnetosphere to the ionosphere and the electric potential from the ionosphere to the magnetosphere. This feedback loop is updated every $4 \mathrm{~s}$.

The magnetic field in the MHD region is separated to a curl-free (dipole) component and perturbed component created by currents external to the Earth (B $=\mathbf{B}_{0}+\mathbf{B 1}(t)$; Tanaka, 1994). In order to make the computations feasible on one polar cap (PC), GUMICS-4 uses temporal subcycling and adaptive Cartesian octogrid. The former reduces the number of MHD computations an order of magnitude while maintaining the local Courant-Friedrichs-Levy constraint (Lions, 2000, pp. 121-151). The latter ensures that whenever there are large gradients, the grid is refined, thus resolving smaller-scale features especially close to boundaries and current sheets.

The region between the MHD magnetosphere and the electrostatic spherical ionosphere is a passive medium where no currents flow perpendicular to the magnetic field. The ionospheric grid is triangular and densest in the auroral oval, while in the polar caps the grid is still rather dense, with about 180 - and $360-\mathrm{km}$ spacing used in the two regions, respectively. External inputs to the ionosphere are the field-aligned current pattern, electron precipitation, and solar extreme ultraviolet ionization. The last two have contribution on the height-integrated Pedersen and Hall conductivities with solar extreme ultraviolet ionization contributing via $10.7-\mathrm{cm}$ solar radio flux (used as a proxy for solar ultraviolet activity) that has a numerical value of $100 \times 10^{-22} \mathrm{~W} / \mathrm{m}^{2}$. Electron precipitation affects the ionospheric electron densities, which are calculated at different altitudes and are used when computing the height-integrated Pedersen and Hall conductivities. The details regarding the ionsopheric part of GUMICS-4 can be found in Janhunen and Huuskonen (1993).

\subsection{The CPCP in GUMICS-4}

Global MHD codes including magnetosphere-ionosphere coupling are generally prone to close excessive amount of electric current through the PC. This leads either to unrealistically large CPCP values and reasonable AE currents or reasonable CPCP values and low AE currents due to unrealistically low Region 2 currents (De Zeeuw et al., 2004). While most of the codes belong to the first category, GUMICS-4 does not overestimate the CPCP and thus produces lower potential values than its contemporaries (Gordeev et al., 2015). GUMICS-4 also produces higher potential values when runs are performed with high spatial resolutions ( $\left.\max 0.25 R_{E}\right)$. This can be seen in Figure 1, which shows the CPCP response to the IMF $E_{Y}$ during 10-day interval using SW data from OMNIweb service (http://omniweb.gsfc.nasa.gov). The details of the GUMICS-4 run executed using $0.5 R_{E}$ maximum resolution can be found in Lakka et al. (2017). The data are binned by IMF $E_{Y}$ with $0.5-\mathrm{mV} / \mathrm{m}$ intervals, and the CPCP is averaged within these bins. For example, a point at $E_{Y}=-1 \mathrm{mV} / \mathrm{m}$ represents averaged CPCP over $-1.25<E_{Y}<-0.75 \mathrm{mV} / \mathrm{m}$. Standard deviations computed for each bin are used as error bars. The CPCP values are relatively low, but the linear response of the CPCP with moderate $E_{Y}(<3 \mathrm{mV} / \mathrm{m})$ during southward IMF (positive $E_{Y}$ ) can be identified. A nonlinear regime is also shown with $E_{Y}>3 \mathrm{mV} / \mathrm{m}$, thus suggesting that the CPCP saturates. Taking into account that CPCP exceeds $5 \mathrm{kV}$ even with northward IMF (negative $E_{Y}$ ), it is apparent that GUMICS-4 predicts the existence of viscous interaction-driven ionospheric potential. We, however, acknowledge that global MHD simulations are unable to correctly model viscous interaction, and hence, it should be noted that in the context of global MHD modeling viscous processes are governed by numerical diffusion.

An interesting feature in Figure 1 is the decrease of the CPCP with increasing $E_{Y}$ driving during moderate $E_{Y}$ and northward IMF (-2 $\left.<E_{Y}<0 \mathrm{mV} / \mathrm{m}\right)$. This is noted earlier by, for example, Bhattarai et al. (2012) and is caused by the relation between viscous and reconnection potentials in such a way that for low $E_{Y}$ viscous potential exceeds its reconnection counterpart. For southward IMF such reduction of the CPCP does not happen, as can be seen in Figure 1.

\subsection{GUMICS Analysis}

We performed five simulations with each having a duration of $5 \mathrm{hr}$. All simulations were initialized by constant SW driving of $2 \mathrm{hr}$ using upstream values equal to those used during the first hour of the actual simulation. 

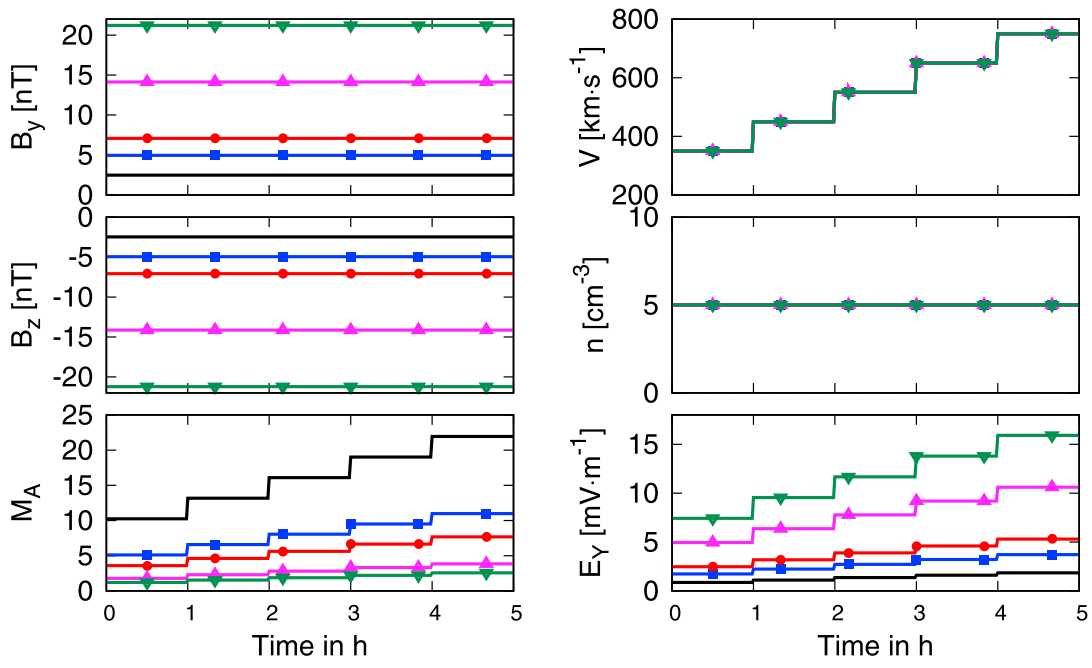

$$
\mathrm{B}=3.5 \mathrm{nT} \longrightarrow \mathrm{B}=7 \mathrm{nT} \rightarrow \mathrm{B}=10 \mathrm{nT} \rightarrow \mathrm{B}=20 \mathrm{nT} \longrightarrow \mathrm{B}=30 \mathrm{nT} \rightarrow
$$

Figure 2. Used solar wind input parameters for each GUMICS-4 simulation run. Left: $B_{Y}, B_{Z}$, and $M_{A}$. Right: $V, n$, and $\left.E_{Y}\right)$. Note that the line plots are identical for $V$ and $n$ and so one color is representing all of them.

The SW velocity $X$ component was increased stepwise from 350 to $750 \mathrm{~km} / \mathrm{s}$ with instant $100-\mathrm{km} / \mathrm{s}$ steps every $1 \mathrm{hr}$. The SW plasma number density and temperature were fixed to statistically rather common values of $5 \mathrm{~cm}^{-3}$ and 30,000 K, respectively (Dimmock et al., 2014). To preserve the $\nabla \cdot \mathbf{B}=0$ condition at the inflow boundary $\left(X=32 R_{E}\right)$, the magnetic field $B_{X}$ component was fixed (set to 0 in the present study). The only input parameter that varied in the simulations was the IMF magnitude, which had five values $(3.5,7,10,20$, and $30 \mathrm{nT}$ ), one for each simulation. The IMF $Y$ and $Z$ components were set equal but of opposite sign (negative $B_{Z}$ and positive $B_{Y}$ ). In other words, the IMF was oriented southward in every simulation with $135^{\circ}$ clock angle. The dipole field tilt angle was set to 0 .

Figure 2 shows how the SW input parameters $\left(B_{Y}, B_{Z}, V_{X}\right.$, and $\left.n\right)$ evolved during the simulation runs. It also shows that such upstream conditions cover SW electric field range from 0.9 to $15.9 \mathrm{mV} / \mathrm{m}$ and Alfvén Mach number range 1.2-22. Details are shown in Table 1.

\section{Results}

\subsection{Ionospheric CPCP Response to Upstream Conditions}

Figures $3 a-3 e$ illustrate the CPCP time evolution computed for the Northern Hemisphere (with zero dipole tilt both hemispheres produce approximately similar results) in the simulations as a function of the IMF $|B|=3.5$, 7, 10, 20, and $30 \mathrm{nT}$. Associated Alfvén Mach numbers for each 1-hr period are also shown. We remind that the upstream plasma flow speed changes every $1 \mathrm{hr}$. If one excludes these transition regions caused by the flow speed change, for the $|B|=3.5-, 7-$, and 10-nT runs (Figures $3 a-3 c$ ), the response of the CPCP to upstream SW is rather stepwise. It should be noted that these runs are also the ones with Alfvén Mach number $M_{A}>4$ everywhere excluding the beginning of the $|B|=10-n T$ run. The response of the CPCP is more complex during runs $|B|=20$ and $30 \mathrm{nT}$ (Figures $3 \mathrm{~d}-3 \mathrm{e}$ ) such that the CPCP even stays fixed for several hours after which it

Table 1

Summary of the Solar Wind $E_{Y}$ and Alfvén Mach Number Ranges Used in the Simulation Runs of the Current Study Classified by the Used IMF $|B|$

\begin{tabular}{lcc}
\hline$|B|^{\mathrm{SW}}(\mathrm{nT})$ & $E_{Y}^{\mathrm{SW}}(\mathrm{mV} / \mathrm{m})$ & $M_{A}$ \\
\hline 3.5 & $0.9-1.9$ & $10.2-22$ \\
7 & $1.7-3.7$ & $5.1-11$ \\
10 & $2.5-5.3$ & $3.6-7.7$ \\
20 & $4.9-10.6$ & $1.8-3.8$ \\
30 & $7.4-15.9$ & $1.2-2.6$ \\
\hline
\end{tabular}



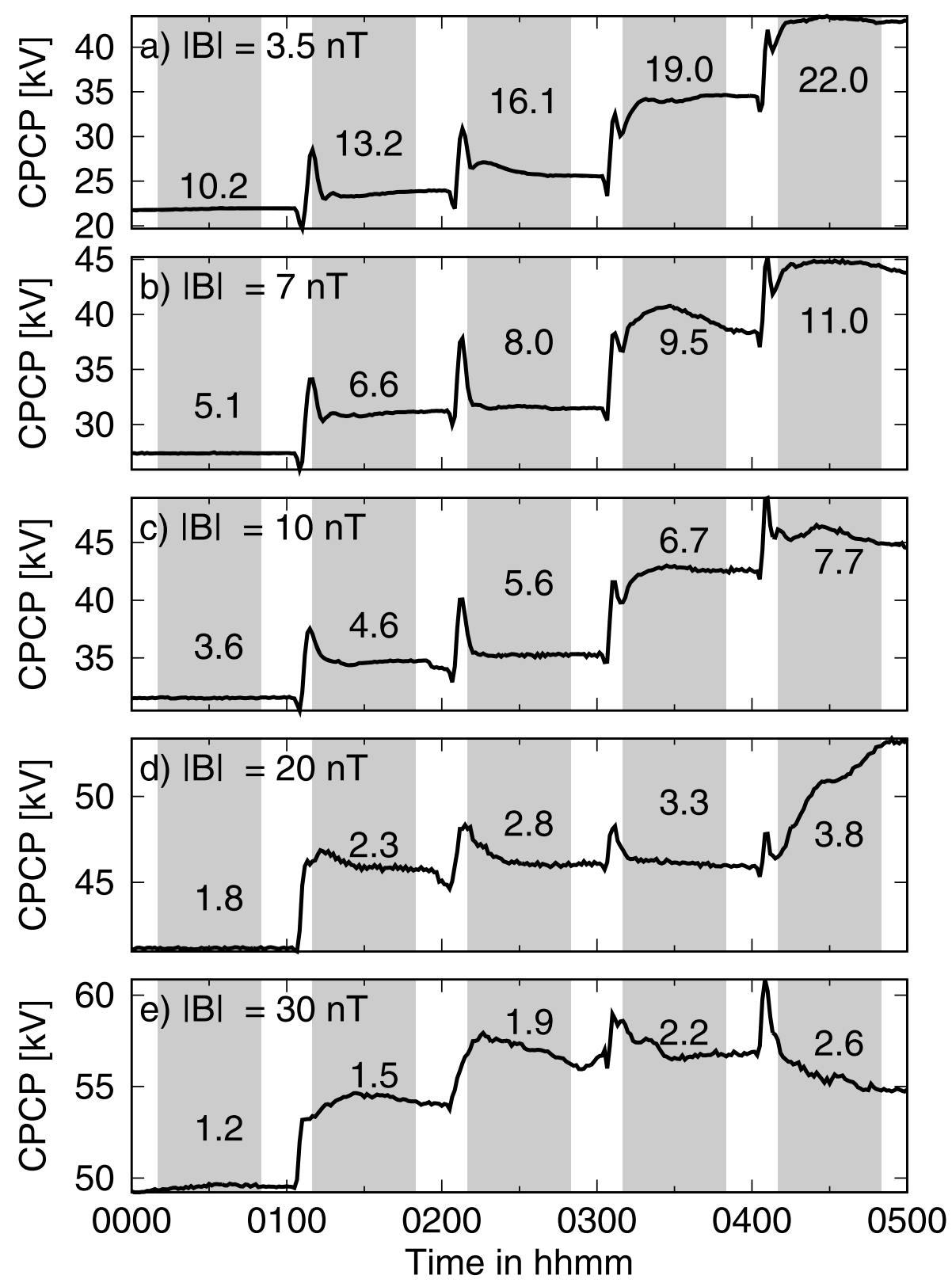

Figure 3. Time series of the cross-polar cap potential (CPCP) computed for the Northern Hemisphere during each simulation run. (a-e) Simulation runs utilizing $|B|=3.5,7,10,20$, and $30 \mathrm{nT}$, respectively. Associated Alfvén Mach numbers are plotted for each 1-hr stage. Highlighted (with gray) 40-min periods were used when computing average potential values for Figure 4 .

starts increasing in Figure 3d, while in Figure 3e the CPCP increases first as a response to the plasma speed changes, stagnates and then decreases during the last 1-hr period.

Figure 4 shows the response of the ionospheric CPCP to the SW $E_{Y}$ such that Figure $4 a$ shows the IMF magnitude, Figure $4 \mathrm{~b}$ the SW speed, and Figure 4c the Alfvén Mach number color coded. Stepwise changing of the upstream flow speed destabilizes the magnetosphere-ionosphere system such that it takes some time for the CPCP to reach a stable value. Therefore, we plot values as 40-min averages computed in the middle of each 1 -hr interval. These 40-min periods are highlighted as gray in Figure 3. It is apparent that this does not necessarily make sure that the effect of the peaks in the CPCP plots indicating destabilized magnetosphere-ionosphere system is neglected everywhere. For the sake of consistency this method is, however, applied for every run 

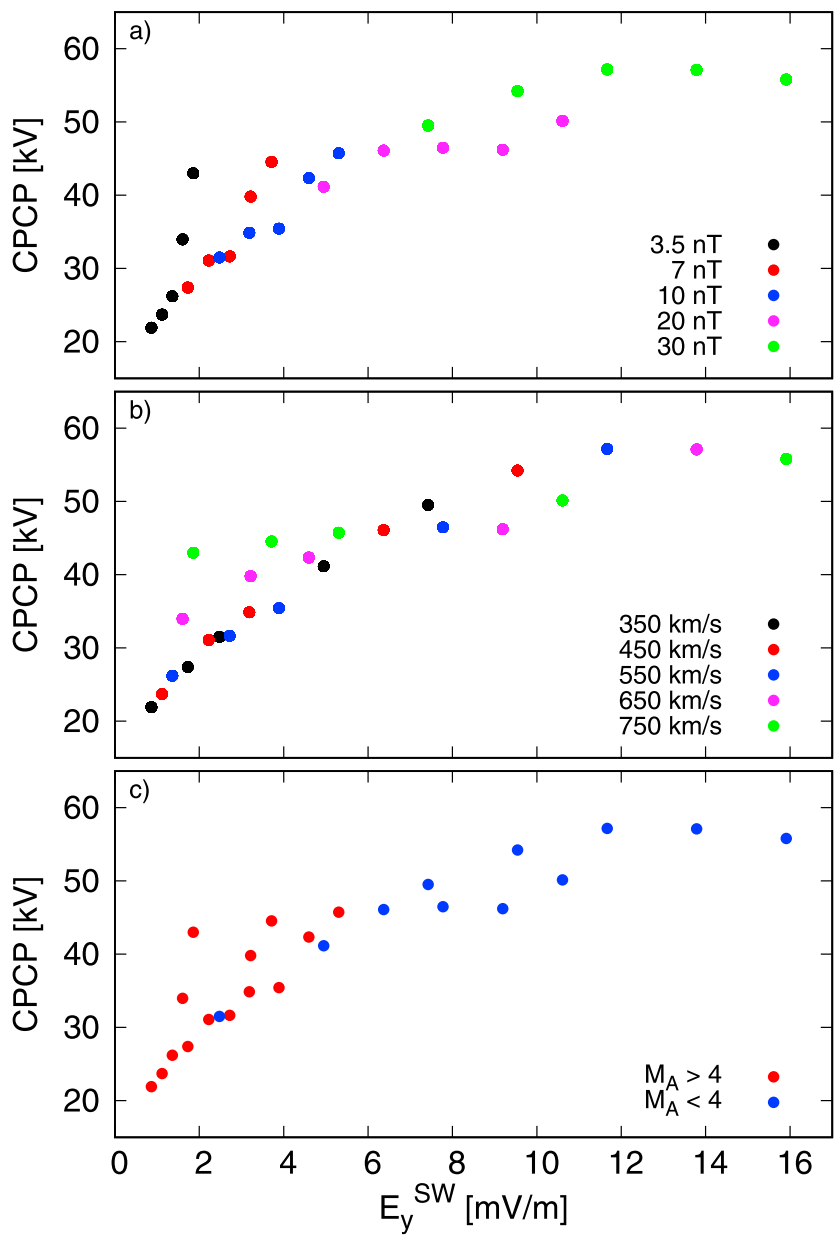

Figure 4. The (averaged over $40 \mathrm{~min}$ ) cross-polar cap potential as a function of the interplanetary magnetic field (IMF) $E_{Y}$ for the five simulation runs with each of them utilizing different IMF magnitude $(3.5,7,10,20$, and $30 \mathrm{nT})$. The used 40-min time periods are highlighted in Figure 3. $(\mathrm{a}-\mathrm{c})$ The magnitudes of the IMF magnitude, the upstream flow speed, and the Alfvén Mach number, respectively. CPCP = cross-polar cap potential.

since in most cases these peaks can be removed from the 40-min averages. In some cases it is also difficult to determine when the system is stabilized.

Since the overall trend of the CPCP response to the upstream conditions in Figure 4 is linear in $E_{Y}$ range from 0 to $4-6 \mathrm{mV} / \mathrm{m}$ and nonlinear from 4 to $6 \mathrm{mV} / \mathrm{m}$ upward the results suggest that the saturation of CPCP starts at $E_{Y}=4-6 \mathrm{mV} / \mathrm{m}$. From this point we are thus referring to the $E_{Y}$ range from 0 to $4-6 \mathrm{mV} / \mathrm{m}$ as linear regime and from 4 to $6 \mathrm{mV} / \mathrm{m}$ upward as nonlinear regime. It should be noted, however, that plasma parameters such as the density have an impact on the numerical value of the saturation $E_{Y}$ (Lopez et al., 2010), and we thus acknowledge that, for example, by using higher number density than the current $5 \mathrm{~cm}^{-3}$ would probably lead to higher saturation $E_{Y}$ value. The point at which the CPCP saturation starts in $E_{Y}$ is therefore not unique but depends on the other upstream parameters as well. This study, however, shows that the saturation electric field $E_{Y}$ with common number density value of $5 \mathrm{~cm}^{-3}$ is $E_{Y}=4-6 \mathrm{mV} / \mathrm{m}$, which is in agreement with previous studies (Shepherd, 2007).

Figure $4 \mathrm{~b}$ suggests that the increase of the CPCP in the linear regime depends on the upstream velocity; for lower velocity values (below $550 \mathrm{~km} / \mathrm{s}$ ) the increase is clearly higher than for higher velocity. The overall trend is consistent with previous studies utilizing statistical (Newell et al., 2008) and numerical (Lopez et al., 2010) tools. The latter suggested that this is caused by the SW flow diversion in the pressure gradient-dominated magnetosheath; faster SW will produce more rapid diversion of the flow around the magnetosphere, and thus, smaller amount of plasma will reach the magnetic reconnection site. 
Table 2

Summary of Additional Simulation Runs Classified by the Used Constant Upstream Parameter (Either $E_{Y}$ or $|B|$ )

\begin{tabular}{lcc}
\hline Constant & $E_{Y}^{\mathrm{SW}}(\mathrm{mV} / \mathrm{m})$ & $|B|^{\mathrm{SW}}(\mathrm{nT})$ \\
\hline$E_{Y}$ & 2 & $4.4-8.1$ \\
$E_{Y}$ & 5 & $11-20$ \\
$E_{Y}$ & 8 & $17-32$ \\
$|B|$ & $7.4-15.9$ & 30 \\
\hline
\end{tabular}

Figure 4c shows that the saturation of the CPCP is strongly dependent on the upstream Alfvén Mach number $M_{A}$ such that $M_{A}$ values below $M_{A}=4$ are found in the nonlinear regime, thus agreeing with Lopez et al. (2010). The dependence of the CPCP saturation on $M_{A}$ is well known, documented both in measurements (Myllys et al., 2016; Wilder et al., 2011) and in simulation results (Lopez et al., 2010). It should be noted, however, that studies based on observational evidence have shown it to happen with large $M_{A}$ values (up to 7.3; (Myllys et al., 2016), 2016). Figure 4 suggests also that in the range $7<E_{Y}<12$ the values of the CPCP differ even if the $E_{Y}$ values would be roughly the same. From Figure $4 a$ it is evident that this scatter of CPCP values appears in simulation runs with $|B|$ of 20 or $30 \mathrm{nT}$. The CPCP values appear to increase when $|B|$ is increased and $|V|$ decreased.

Figures $3 a-3 c$ show that there is a clear step change with $3.5<|B|<10 \mathrm{nT}$ suggesting that the response of the CPCP to upstream changes (SW velocity increases from 350 to $750 \mathrm{~km} / \mathrm{s}$ ) is linear when $3.5<|B|<10 \mathrm{nT}$. Figure 4a suggests the same: data points with $|B|<10 \mathrm{nT}$ are in the linear regime. Figures $3 \mathrm{~d}$ and $3 \mathrm{e}$ show that the CPCP time evolution is more complex, the value of the CPCP is approximately fixed from 0100 to 0400 in panel (d) and from 0200 to 0500 in panel (e). In panel (d) the CPCP starts increasing at 0400 after a period of fixed value and it coincides with $M_{A}$ value increasing from 3.3 to 3.8. Moreover, the CPCP increases from the first (0000-0100) to the second (0100-0200) hour in $|B|=20-\mathrm{nT}$ run as well as from the first to third (0000-0300) in $|B|=30-n T$ run. At the same time $M_{A}$ changes from $M_{A}<2$ to $M_{A}>2$.

\subsection{The Effect of Low $M_{A}$ During Initialization}

Figure 4 illustrates some notable features covered in section 3.1. In the linear regime the increase of the CPCP appears to depend on the SW speed, and in the saturation regime the same IMF $E_{Y}$ produces different CPCP values when $7 \mathrm{mV} / \mathrm{m}<E_{Y}<12 \mathrm{mV} / \mathrm{m}$. Since simulation initial conditions may play a significant role in global MHD simulations (Lakka et al., 2017) and since some of the runs have rather large IMF magnitude during initialization, we test whether the use of large IMF $|B|$ during simulation initialization has an effect on these features. To that end, an additional set of runs was executed with constant IMF $E_{Y}(2,5$, and $8 \mathrm{mV} / \mathrm{m})$ and varying upstream flow speed (350-650 km/s) and IMF magnitude (depending on simulation $4.3-32.3 \mathrm{nT}$ ). To initialize constant $E_{Y}$ runs, the final state of $|B|=10 \mathrm{nT}$ was used to represent low (in the context of the current study) $M_{A}$ (7.7) and sufficiently low level of driving. Furthermore, switching between different upstream conditions was not instantaneous but linear with each upstream condition switch lasting 10 min. Moreover, to make direct comparison between lower and higher driving conditions during simulation initialization, the run with IMF $|B|=30-\mathrm{nT}$ was run again with changing the upstream flow speed in reversed order, from 750 to $350 \mathrm{~km} / \mathrm{s}$. The details of all four simulations are listed in Table 2.

Figure 5 shows results from Figure 4 (filled circles) together with constant IMF $E_{Y}$ runs and reversed IMF $|B|=$ 30-nT run (filled and half-filled rectangles). As in the case of Figure 4, the points are differentiated by IMF $|B|$ (Figure 5a), SW flow speed (Figure 5b), and SW Alfvén Mach number (Figure 5c). The results from constant $E_{Y}$ runs blend in remarkably well. For example, data from $E_{Y}=2 \mathrm{mV} / \mathrm{m}$ show similar dependence on the $\mathrm{SW}$ flow speed as the results from the simulations in which IMF $E_{Y}$ is not fixed.

By looking at the nonlinear regime, it is apparent that the CPCP depends on the evolution of $M_{A}$ during the simulation run; running the $|B|=30-\mathrm{nT}$ run with the upstream flow speed changes reversed and thus starting with higher $M_{A}$ creates lower CPCP values. The difference in the CPCP between increasing and decreasing SW speed in the $|B|=30-\mathrm{nT}$ simulations is highest at lower end of the $E_{Y}$ range, while the difference becomes negligible at the higher end. Since both $|B|=20$ - and $|B|=30-\mathrm{nT}$ runs start with small $M_{A}$ values, we repeated the $|B|=20-\mathrm{nT}$ run with $|V|$ reversed and obtained different CPCP values as well, with increased CPCP in the nonlinear regime. On the other hand, results from $E_{Y}=8-\mathrm{mV} / \mathrm{m}$ run repeat behavior 


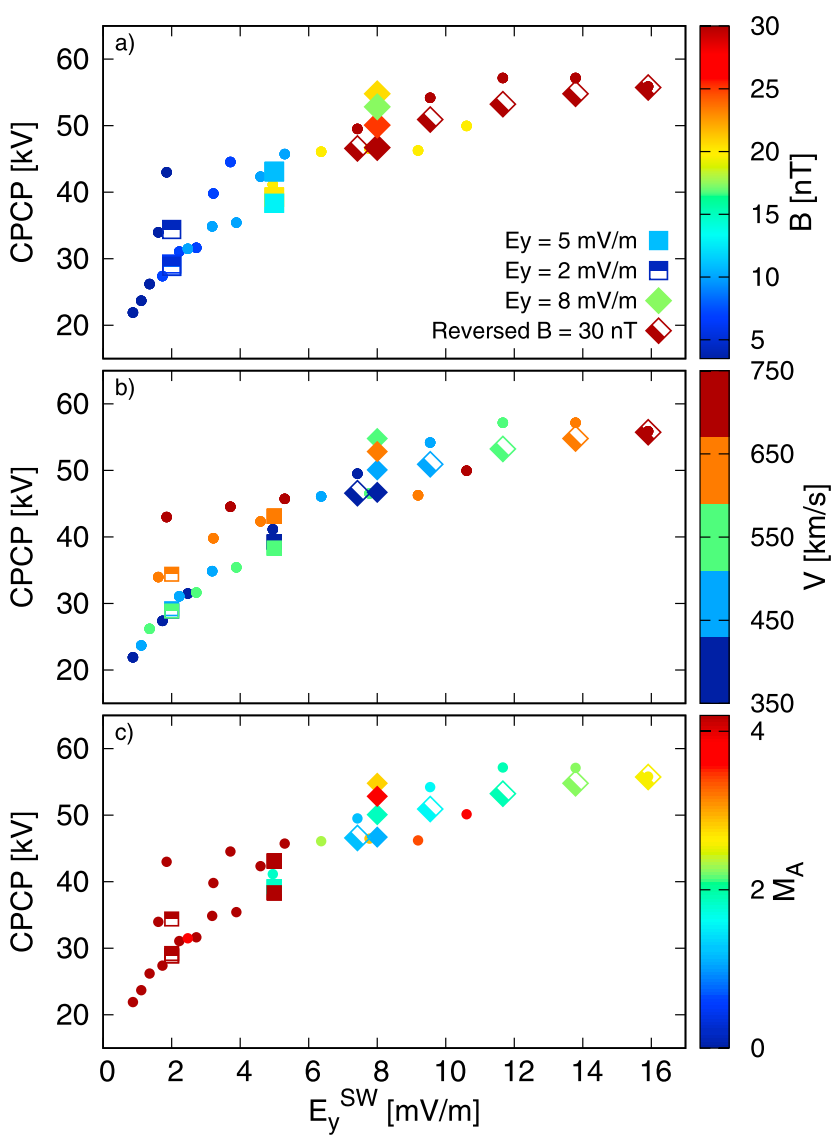

Figure 5. The cross-polar cap potential (CPCP) as a function of the interplanetary magnetic field (IMF) $E_{Y}$ for the five simulations shown in Figure 4 (filled circles) accompanied by the additional runs with either IMF $E_{Y}$ or $|B|$ fixed (filled or half-filled rectangles). (a-c) The magnitudes of the IMF magnitude, the upstream flow speed and the Alfvén Mach number, respectively.

analogous to previous runs; same IMF $E_{Y}$ values can produce different CPCP values. However, while comparing the $|B|=20$ - and $|B|=30-n T$ runs with increasing and decreasing speed, it is apparent that increasing $\mathrm{IMF}|B|$ and decreasing SW speed resulted in increasing CPCP, while $E_{Y}=8-\mathrm{mV} / \mathrm{m}$ run manifests the opposite; decreasing IMF magnitude and increasing upstream flow speed results in increase in CPCP (Figures 5 a and $5 \mathrm{~b}$ ). It should be noted, however, that due to lower $M_{A}$ during the initialization, the $E_{Y}=8-\mathrm{mV} / \mathrm{m}$ run is more reliable than the constant $|B|$ runs. We conclude that $E_{Y}=8-\mathrm{mV} / \mathrm{m}$ run correctly captures the response of CPCP to IMF $E_{Y}$ such that decreasing $|B|$ and increasing $|V|$ results in an increase in CPCP. The reason that the $|B|=20$ and $|B|=30 \mathrm{nT}$ with increasing speed runs fail to do this is the too low $M_{A}$ during the initialization.

\section{Discussion}

In this paper we study the response of the ionospheric CPCP to the upstream changes during high and low SW driving. We used artificial SW data and combined both high and low IMF magnitudes with high and low SW Alfvén Mach numbers by altering the SW speed. We conclude that the CPCP response is linear (nonlinear) below (above) $E_{Y}^{\mathrm{SW}}=4-6 \mathrm{mV} / \mathrm{m}$, which agree fairly well with observational values that range between 0.5 and $10 \mathrm{mV} / \mathrm{m}$ depending on the upstream conditions (Russell et al., 2001; Shepherd, 2007). GUMICS-4 also produces considerably lower CPCP values when lower spatial resolution is used. This is evident when comparing Figures 1 and 4.

Figure 6 shows statistical measurements of both AE and PC indices from 1963 to 2016 using the OMNIWeb service (http://omniweb.gsfc.nasa.gov/). AE index is a measure of auroral zone magnetic activity produced by enhanced currents (Davis \& Sugiura, 1966), while PC index measures magnetic activity in the polar cap (Troshichev et al., 1988). The data are divided into five speed ranges (less than 450, 450-550, 550-650, 

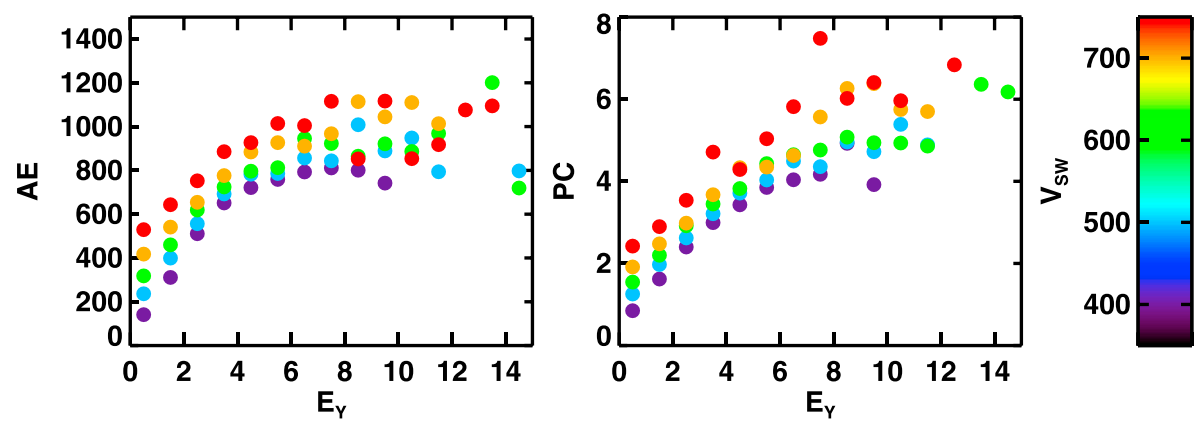

Figure 6. Auroral electrojet (AE) and polar cap (PC) index as a function of the upstream electric field $Y$ component from 1963 to present day. The data are retrieved from OMNIWeb service (http://omniweb.gsfc.nasa.gov/) and divided into five speed ranges (less than 450, 450-550, 550-650, 650-750, and more than $750 \mathrm{~km} / \mathrm{s}$ ) and binned by parallel (to the geomagnetic field) electric field component $E_{Y}$ with $1-\mathrm{mV} / \mathrm{m}$ intervals. For each bin, the mean value of $\mathrm{AE}$ was computed.

$650-750$, and more than $750 \mathrm{~km} / \mathrm{s}$ ) and binned by electric field component $E_{Y}$ with $1-\mathrm{mV} / \mathrm{m}$ intervals. For each bin, the mean value of $A E$ and $P C$ was computed. Since geomagnetic indices such as AE estimate the energy dissipation caused by the $S W$ into the magnetosphere, the response of $A E$ to the upstream conditions should agree with the CPCP response. Indeed, Figure 6 shows that over several decades the response of both $\mathrm{AE}$ and $\mathrm{PC}$ to the $Y$ component of IMF $E$ is similar to that found in this study (Figure 4) such that the larger SW speed produces more steep increase of $A E$ and $P C$ with $E_{Y}$ values lower than at saturation point, which is $E_{Y}>6 \mathrm{mV} / \mathrm{m}$.

We note that the actual numerical value of saturation $E_{Y}^{\mathrm{SW}}$ depends also on other parameters such as density (Lopez et al., 2010), which in our simulations had a fixed value of $5 \mathrm{~cm}^{-3}$. We also acknowledge that GUMICS-4 produces generally lower CPCP values than other global MHD codes (Gordeev et al., 2015) due to different closure of the electric current through the PC; due to nonexistent Region 2 currents, relatively large amount of current closes through the PC (Janhunen et al., 2012). Moreover, Region 1 currents are spread over a large area in GUMICS-4. As a consequence, while most other codes produce unrealistically large CPCP values and reasonable AE currents, GUMICS-4 produces reasonable CPCP values and unrealistically low $A E$ currents. Furthermore, magnetic reconnection is described differently in the various codes. For example, a Block-Adaptive-Tree-Solarwind-Roe-Upwind-Scheme (Powell et al., 1999) code user can apply a resistive point in the dayside reconnection region to ensure that the reconnection rate is calculated correctly at the dayside. In the absence of such resistive point, the reconnection rate is governed by numerical diffusion in solving the MHD equations (Borovsky et al., 2009). Despite these differences, this study agrees well with previous numerical studies. For example, Figure $4 \mathrm{~b}$ suggests that the increase of the CPCP in the linear regime $\left(E_{Y}^{\mathrm{SW}}<4-6 \mathrm{mV} / \mathrm{m}\right.$ ) increases depending on the upstream SW speed: for lower speed values (below $550 \mathrm{~km} / \mathrm{s}$ ) the slope of the curve is larger than for higher speed values consistent with Lopez et al. (2010) who used the Lyon-Fedder-Mobarry (LFM; Lyon et al., 2004) code, and with a statistical study by Newell et al. (2008). Similar results was obtained by Bhattarai et al. (2012) for northward IMF. Lopez et al. (2010) suggest that the effect is caused by the SW flow diversion in the pressure gradient-dominated magnetosheath; a faster SW will produce a more rapid diversion of the flow around the magnetosphere, and thus, a smaller amount of flow will reach the magnetic reconnection region.

Another feature shown in Figure 4 is the dependence of the CPCP saturation on the upstream Alfvén Mach number $M_{A}$ such that saturation starts when $M_{A}<4$. Lavraud and Borovsky (2008) suggest that the overall magnetosheath plasma beta $\left(p / p_{B}\right.$, where $p$ is the plasma pressure and $p_{B}$ the magnetic pressure) decreases below 1 when the Alfvén Mach number decreases below 4 . Under these conditions the force balance of the magnetosheath changes and the $\mathbf{J} \times \mathbf{B}$ force dominates the MHD momentum equation:

$$
\rho\left(\frac{\partial}{\partial t}+\mathbf{v} \cdot \nabla\right) \mathbf{v}=\mathbf{J} \times \mathbf{B}-\nabla
$$

over fluid stresses ( $\nabla p$ in the MHD momentum equation) in the magnetosheath. In the above equation $\rho$ is the density, $\mathbf{v}$ the velocity, $\mathbf{J}$ the current density, $\mathbf{B}$ the magnetic field, and $p$ the pressure. Figure 7 shows 
a) $M_{A}=2.8$
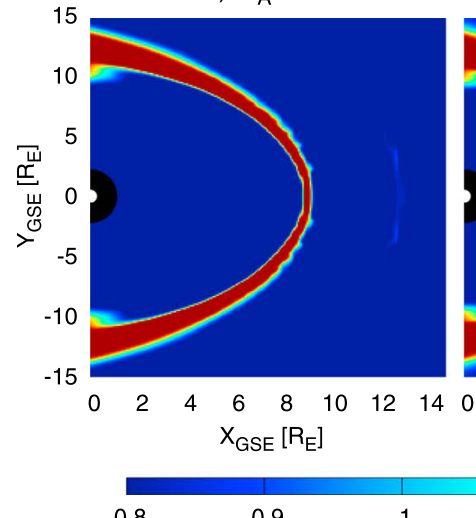

0.9 b) $\mathrm{M}_{\mathrm{A}}=3.3$

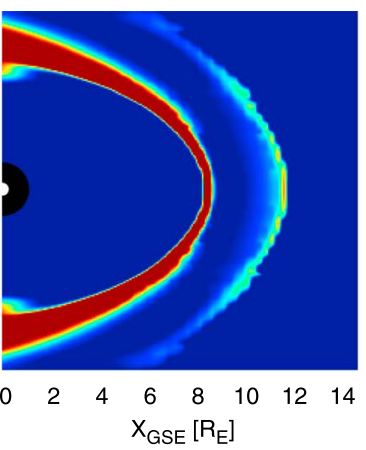

c) $\mathrm{M}_{\mathrm{A}}=3.8$

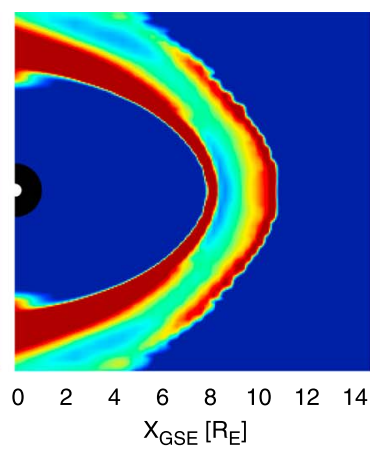

1.3
1.4

Figure 7. Plasma beta surface plot during the $|B|=20-n T$ simulation run at 2.50 (panel a), 3.50 (panel b), and 4.50 (panel c) when the upstream Alfvén Mach number increases from 2.8 to 3.8 .

a collection of surface plots with plasma beta color coded during the $|B|=20-n T$ simulation run. Panels (a) - (c) correspond to upstream conditions of $|\mathrm{V}|=550,650$, and $750 \mathrm{~km} / \mathrm{s}$. The panels show time instances of 0250 (panel a), 0350 (panel b), and 0450 (panel c). During this interval, the upstream Alfvén Mach number increases from 2.8 to 3.8. Figure 7 shows that the overall plasma beta parameter changes from below 1 to above 1 in the magnetosheath suggesting that when $M_{A}$ approaches 4 , the magnetosheath force balance undergoes a transition from the $\mathbf{J} \times \mathbf{B}$-dominated to pressure gradient-dominated magnetosheath. Lopez et al. (2010) state that when the magnetic forces are enhanced in the magnetosheath at the expense of pressure gradient forces, the main contributor to slowing of the SW is the magnetic shear instead of fluid stresses. Because of magnetic shear, a current flows along the bow shock as Ampere's law provides an outward force slowing the SW. As a consequence the SW flow diverts around the magnetosphere in a different manner, and the amount of flow entering the reconnection region is reduced thus reducing also the CPCP. That is, to say, plasma flow streamlines are diverted away from the merging region in the dayside magnetopause. To see if this happens in our simulations, we plot plasma beta parameter in Figure 8 in the equatorial plane for two simulation scenarios with different characteristics: low Alfvén Mach number (22 in Figure 8a) and high Alfvén Mach number (3.8 in Figure 8b). Plasma flow streamlines are also shown. They begin in the SW near the equatorial plane at $Z_{\mathrm{GSE}}=0.2 R_{E}, \pm Y_{\mathrm{GSE}}=7,6.25,5.5,4.75,4,3.25,2.5,1.75$, and $1 R_{E}$. Also shown are magnetic field lines that have one foot point in the ionosphere and the other in the SW, thus showing the width of the merging region. Both Figures $8 \mathrm{a}$ and $8 \mathrm{~b}$ are snapshots at 0450 of simulation runs $|B|=3.5$ and $20 \mathrm{nT}$, respectively, and thus, the SW speed is $750 \mathrm{~km} / \mathrm{s}$ in both cases. Comparing Figures $8 \mathrm{a}$ and $8 \mathrm{~b}$, it is obvious that the magnetosheath plasma beta is above 1 (deep red) everywhere in the 3.5-nT case, while in the 20-nT case transition from above 1 to below 1 is about to take place (green and yellow). Actually, Figure $8 \mathrm{~b}$ is basically identical to Figure $7 c_{\text {, }}$ with only plasma streamlines and magnetic field lines added, and thus, the magnetosheath plasma beta goes below 1 as the SW Alfvén Mach number reaches 2.8 (Figures 7a-7c).

Figure 8 shows that the number of plasma streamlines that intersect the merging region is larger in the 3.5-nT case than in the 20-nT case. Thus, the amount of SW plasma reaching the merging region shrinks as the Alfvén Mach number diminishes from 22 to 3.8 and the magnetosheath plasma beta transit from above 1 to below 1 takes place. This eventually reduces the CPCP, as was reported also by Lopez et al. (2010) and Lavraud and Borovsky (2008). We have used only one SW usptream density value $\left(5 \mathrm{~cm}^{-3}\right)$ and thus not tested if the CPCP saturation dependence of the upstream Alfvén Mach number is valid for other density values, too. We have, however, studied an ICME event that occurred during 14 July 2012 with GUMICS-4 and will report the results in an upcoming paper. This particular event features fluctuating plasma number density, very low $M_{A}$, and strongly southward IMF. With such upstream conditions saturation is still dependent on $M_{A}$ even if the SW plasma number density varies. 
a) $0450 \mathrm{~B}_{m a g}=3.5 \mathrm{nT}$

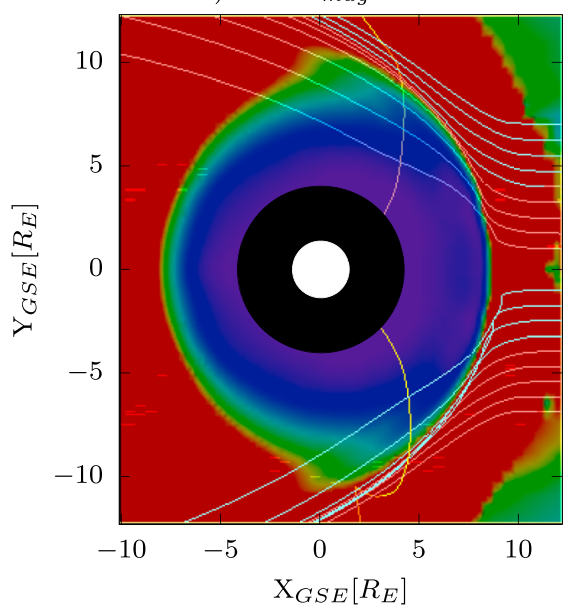

b) $0450 \mathrm{~B}_{m a g}=20 \mathrm{nT}$

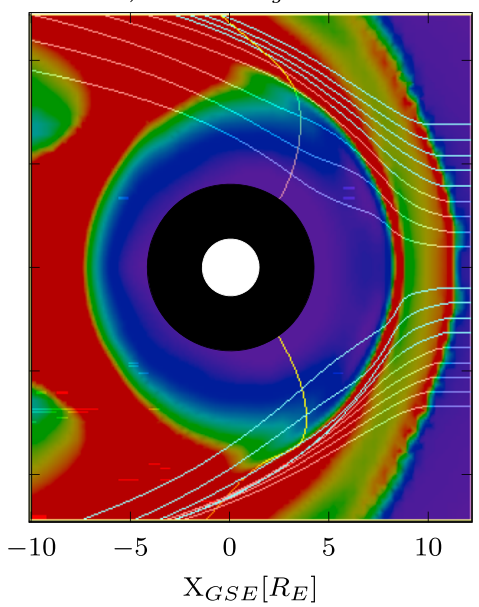

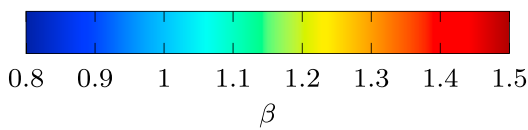

Figure 8. Plasma beta in the equatorial plane during the $|B|=3.5$ - and 20-nT simulation runs at 0450, when solar wind speed was $750 \mathrm{~km} / \mathrm{s}$. Plasma flow streamlines begin in the solar wind near the equatorial plane at $\pm Y_{\mathrm{GSE}}=7,6.25,5.5$, $4.75,4,3.25,2.5,1.75$, and $1 R_{E}$. Also shown are magnetic field lines that have one foot point in the ionosphere and the other in the solar wind thus showing the width of the merging region. Note that the figures are cut planes of 3-D plots.

Figures $3 d, 3 e$, and $4 c$ show that after the magnetosheath force balance has changed, that is, when the Alfvén Mach number becomes significantly lower than 4, the CPCP value starts decreasing even more. This is shown, for example, in Figure 3d examining the $|B|=20$-nT run from 0500 to 0000 in simulation physical time; the numerical value of the CPCP is fixed to approximately $47 \mathrm{kV}$ during $0400-0100$. At the same time, $M_{A}$ decreases from 3.3 to 2.3. When $M_{A}$ decreases from 2.3 to 1.8 , the CPCP is decreased to $\sim 40 \mathrm{kV}$. Similar development of the CPCP can be seen in Figure 3 e for the $|B|=30-n T$ run; when $M_{A}$ decreases from 1.9 to 1.2 during $0300-0000$, the CPCP reduces from 57 to below $50 \mathrm{kV}$. Since the plasma beta in the magnetosheath is already well below 1, models such as the force balance model (Lopez et al., 2010) cannot explain this decrease. Instead, the impact of this low Alfvén Mach number on the CPCP saturation can be explained by a process external to the Earth's space environment. One such process related to very low upstream $M_{A}$ values is the formation of Alfvén wings (Ridley, 2007). When the IMF encounters an obstacle, the field lines start to bend and Alfvén waves are launched along the field lines with a speed of $V_{A}=B / \sqrt{(}\left(\mu_{0} \rho\right)$. Since the plasma is still flowing with a velocity $V$ and diverting around the obstacle, two tubes with different flow characteristics are created above and below the obstacle. These tubes are called Alfvén wings. Within the wing structure the electric field does not change significantly as the SW driving increases, and hence, the reconnection potential would be saturated, thus leading to saturated ionospheric potential as a consequence. Ridley (2007) suggested originally that the $M_{A}$ values related to the Alfvén wing model are very small, even close to unity. However, later works by, for example, Wilder et al. (2015) have shown that the Alfvén wings start becoming more prominent in the vicinity the Earth when the $M_{A}$ is below 3 .

It is not straightforward to argue that the Alfvén wing model is responsible for the reduction of the CPCP with very low upstream $M_{A}$ values due to lack of observational evidence of such process taking place in the Earth's space environment, This is because the upstream conditions needed (very low $M_{A}$ ) occur very rarely. First paper offering observational evidence by Chané et al. (2012) shows a case where the upstream $M_{A}$ was low due to low density rather than high IMF magnitude; thus, those results are not directly comparable to the results of this paper. One could also argue that since very low upstream $M_{A}$ during simulation initialization causes differences in the CPCP of several kilovolts (as is shown in Figure 5), Alfvén wings are due to incorrect initialization. However, this is unlikely, as the wings have been observed in multiple studies utilizing global MHD models (e.g., Ridley, 2007; Wilder et al., 2015). We note that papers on global MHD codes often fail to elaborate on the used initialization method, and thus, the upstream Alfvén Mach number during initialization is not documented. 


\section{Conclusions}

We conclude the following:

1. In the GUMICS-4 simulation, the response of the CPCP to the SW electric field $Y$ component $E_{Y}$ is linear (nonlinear) below (above) $E_{Y}^{S W}=4-6 \mathrm{mV} / \mathrm{m}$ when the SW number density is $5 \mathrm{~m}^{-3}$. GUMICS-4 reproduces the CPCP response to the upstream conditions similarly to other MHD codes even if there are notable differences between the codes.

2. The ionospheric response is strongly affected by the upstream $M_{A}$ value such that saturation of the CPCP occurs only when $M_{A}<4$ and the plasma beta is below 1 . Such a magnetosheath conditions can lead to altered plasma flow pattern that can trigger the saturation of CPCP as suggested by Lavraud and Borovsky (2008) and Lopez et al. (2010).

3. There is another drop in the CPCP when $M_{A}$ decreases close to 2 , and the force balance of the magnetosheath is dominated by magnetic forces. This can be a sign of a process triggered by the upstream changes contributing to the saturation of the CPCP.

4. Initializing a global MHD simulation with very low upstream $M_{A}$ can cause differences in the CPCP value order of several kilovolts, thus emphasizing the importance of initialization in setting up the magnetosphere-ionosphere coupling in the simulation.

\section{Acknowledgments}

We thank the NASA/GSFC Space

Physics Data Facility for maintaining and developing the OMNIWeb and OMNI data. This work has been supported by Academy of Finland grants 1267087,288472 , and 310444 The calculations presented above were performed using computer resources within the Aalto University School of Science "Science-IT" project.

\section{References}

Axford, W. I., \& Hines, C. O. (1961). A unifying theory of high-latitude geophysical phenomena and geomagnetic storms. Canadian Journal of Physics, 39(10), 1433-1464. https://doi.org/10.1139/p61-172

Bhattarai, S. K., Lopez, R. E., Bruntz, R., Lyon, J. G., \& Wiltberger, M. (2012). Simulation of the polar cap potential during periods with northward interplanetary magnetic field. Journal of Geophysical Research, 117, A04219. https://doi.org/10.1029/2011JA017143

Borovsky, J. E., Lavraud, B., \& Kuznetsova, M. M. (2009). Polar cap potential saturation, dayside reconnection, and changes to the magnetosphere. Journal of Geophysical Research, 114, A03224. https://doi.org/10.1029/2009JA014058

Chané, E., Saur, J., Neubauer, F. M., Raeder, J., \& Poedts, S. (2012). Observational evidence of Alfvén wings at the Earth. Journal of Geophysical Research, 117, A09217. https://doi.org/10.1029/2012JA017628

Crooker, N. U. (1988). Mapping the merging potential from the magnetopause to the ionosphere through the dayside cusp. Journal of Geophysical Research, 93(A7), 7338-7344. https://doi.org/10.1029/JA093iA07p07338

Davis, T. N., \& Sugiura, M. (1966). Auroral electrojet activity index AE and its universal time variations. Journal of Geophysical Research, 71(3), 785-801. https://doi.org/10.1029/JZ071i003p00785

De Zeeuw, D. L., Sazykin, S., Wolf, R. A., Gombosi, T. I., Ridley, A. J., \& Tóth, G. (2004). Coupling of a global MHD code and an inner magnetospheric model: Initial results. Journal of Geophysical Research, 109, A12219. https://doi.org/10.1029/2003JA010366

Dimmock, A. P., Nykyri, K., \& Pulkkinen, T. I. (2014). A statistical study of magnetic field fluctuations in the dayside magnetosheath and their dependence on upstream solar wind conditions. Journal of Geophysical Research: Space Physics, 119, 6231-6248. https://doi.org/10.1002/2014JA020009

Dungey, J. W. (1961). Interplanetary magnetic field and the auroral zones. Physical Review Letters, 6, 47-48. https://doi.org/10.1103/PhysRevLett.6.47

Gordeev, E., Sergeev, V., Honkonen, I., Kuznetsova, M., Rastätter, L., Tóth, G., et al. (2015). Assessing the performance of community-available global MHD models using key system parameters and empirical relationships. Space Weather, 13, 868-884 https://doi.org/10.1002/2015SW001307

Hill, T. (1994). Theoretical models of polar-cap convection under the influence of a northward interplanetary magnetic field. Journal of Atmospheric and Terrestrial Physics, 56(2), 185-194. https://doi.org/10.1016/0021-9169(94) 90029-9

Hill, T. W., Dessler, A. J., \& Wolf, R. A. (1976). Mercury and Mars: The role of ionospheric conductivity in the acceleration of magnetospheric particles. Geophysical Research Letters, 3(8), 429-432. https://doi.org/10.1029/GL003i008p00429

Janhunen, P., \& Huuskonen, A. (1993). A numerical ionosphere-magnetosphere coupling model with variable conductivities. Journal of Geophysical Research, 98(A6), 9519-9530. https://doi.org/10.1029/92JA02973

Janhunen, P., Palmroth, M., Laitinen, T., Honkonen, I., Juusola, L., FacskÃș, G., \& Pulkkinen, T. (2012). The GUMICS-4 global $\{\mathrm{MHD}\}$ magnetosphere-ionosphere coupling simulation. Journal of Atmospheric and Solar-Terrestrial Physics, 80, 48-59. https://doi.org/10.1016/j.jastp.2012.03.006

Koustov, A. V., Khachikjan, G. Y., Makarevich, R. A., \& Bryant, C. (2009). On the SuperDARN cross polar cap potential saturation effect. Annales Geophysicae, 27, 3755-3764. https://doi.org/10.5194/angeo-27-3755-2009

Kubota, Y., Nagatsuma, T., Den, M., Tanaka, T., \& Fujita, S. (2017). Polar cap potential saturation during the Bastille Day storm event using global MHD simulation. Journal of Geophysical Research: Space Physics, 122, 4398-4409. https://doi.org/10.1002/2016JA023851

Lakka, A., Pulkkinen, T. I., Dimmock, A. P., Osmane, A., Honkonen, I., Palmroth, M., \& Janhunen, P. (2017). The impact on global magnetohydrodynamic simulations from varying initialisation methods: Results from GUMICS-4. Annales Geophysicae, 35(4), $907-922$. https://doi.org/10.5194/angeo-35-907-2017

Lavraud, B., \& Borovsky, J. E. (2008). Altered solar wind-magnetosphere interaction at low Mach numbers: Coronal mass ejections. Journal of Geophysical Research, 113, A00B08. https://doi.org/10.1029/2008JA013192

Lions, J. L. (2000). Handbook of numerical analysis. Solution of equations in Rn (Part 3), techniques of scientific computing (Part3) (Vol. 7). North-Holland: Elsevier Science \& Techno.

Lockwood, M. (1991). On flow reversal boundaries and transpolar voltage in average models of high-latitude convection. Planetary and Space Science, 39(3), 397-409. https://doi.org/10.1016/0032-0633(91)90002-R

Lockwood, M., \& Smith, M. F. (1992). The variation of reconnection rate at the dayside magnetopause and cusp ion precipitation. Journal of Geophysical Research: Space Physics, 97(A10), 14,841-14,847. https://doi.org/10.1029/92JA01261

Lopez, R. E., Bruntz, R., Mitchell, E. J., Wiltberger, M, Lyon, J. G., \& Merkin, V. G. (2010). Role of magnetosheath force balance in regulating the dayside reconnection potential. Journal of Geophysical Research, 115, A12216. https://doi.org/10.1029/2009JA014597 
Lyon, J. G., Fedder, J. A., \& Mobarry, C. M. (2004). The Lyon-Fedder-Mobarry (LFM) global MHD magnetospheric simulation code. Journal of Atmospheric and Solar-Terrestrial Physics, 66, 1333-1350. https://doi.org/10.1016/j.jastp.2004.03.020

Milan, S. E. (2004). Dayside and nightside contributions to the cross polar cap potential: Placing an upper limit on a viscous-like interaction. Annales Geophysicae, 22(10), 3771-3777. https://doi.org/10.5194/angeo-22-3771-2004

Myllys, M., Kilpua, E., Lavraud, B., \& Pulkkinen, T. I. (2016). Solar wind-magnetosphere coupling efficiency during ejecta and sheath-driven geomagnetic storms. Journal of Geophysical Research: Space Physics, 121, 4378-4396. https://doi.org/10.1002/2016JA022407

Nagatsuma, T. (2004). Conductivity dependence of cross-polar potential saturation. Journal of Geophysical Research, 109, A04210. https://doi.org/10.1029/2003JA010286

Newell, P. T., Sotirelis, T., Liou, K., \& Rich, F. J. (2008). Pairs of solar wind-magnetosphere coupling functions: Combining a merging term with a viscous term works best. Journal of Geophysical Research, 113, A04218. https://doi.org/10.1029/2007JA012825

Nishida, A. (1968). Coherence of geomagnetic DP 2 fluctuations with interplanetary magnetic variations. Journal of Geophysical Research, 73(17), 5549-5559. https://doi.org/10.1029/JA073i017p05549

Powell, K. G., Roe, P. L., Linde, T. J., Gombosi, T. I., \& Zeeuw, D. L. D. (1999). A solution-adaptive upwind scheme for ideal magnetohydrodynamics. Journal of Computational Physics, 154(2), 284-309. https://doi.org/10.1006/jcph.1999.6299

Pulkkinen, T. I., Dimmock, A. P., Lakka, A., Osmane, A., Kilpua, E., Myllys, M., et al. (2016). Magnetosheath control of solar wind-magnetosphere coupling efficiency. Journal of Geophysical Research: Space Physics, 121, 8728-8739. https://doi.org/10.1002/2016JA023011

Reiff, P. (1986). Solar wind control of the polar-cap voltage. In Solar wind-magnetosphere coupling; Proceedings of the Chapman Conference (pp. 453-476). Pasadena, CA.

Reiff, P. H., Spiro, R. W., \& Hill, T. W. (1981). Dependence of polar cap potential drop on interplanetary parameters. Journal of Geophysical Research, 86(A9), 7639-7648. https://doi.org/10.1029/JA086iA09p07639

Ridley, A. J. (2005). A new formulation for the ionospheric cross polar cap potential including saturation effects, 23(11), 3533-3547.

Ridley, A. J. (2007). Alfvén wings at Earth's magnetosphere under strong interplanetary magnetic fields. Annales Geophysicae, 25(2), 533-542. https://doi.org/10.5194/angeo-25-533-2007

Ridley, A. J., Gombosi, T. I., \& Dezeeuw, D. L. (2004). lonospheric control of the magnetosphere: Conductance, 22(2), $567-584$

Russell, C. T., Luhmann, J. G., \& Lu, G. (2001). Nonlinear response of the polar ionosphere to large values of the interplanetary electric field. Journal of Geophysical Research, 106(A9), 18,495-18,504. https://doi.org/10.1029/2001JA900053

Shepherd, S. G. (2007). Polar cap potential saturation: Observations, theory, and modeling. Journal of Atmospheric and Solar-Terrestrial Physics, 69(3), 234-248.

Siscoe, G. L., Erickson, G. M., Sonnerup, B. U. Ö., Maynard, N. C., Schoendorf, J. A., Siebert, K. D., et al. (2002). Hill model of transpolar potential saturation: Comparisons with MHD simulations. Journal of Geophysical Research: Space Physics, 107(A6), 1075. https://doi.org/10.1029/2001JA000109

Siscoe, G. L., \& Huang, T. S. (1985). Polar cap inflation and deflation. Journal of Geophysical Research, 90(A1), $543-547$. https://doi.org/10.1029/JA090iA01p00543

Tanaka, T. (1994). Finite volume TVD scheme on an unstructured grid system for three-dimensional MHD simulation of inhomogeneous systems including strong background potential fields. Journal of Computational Physics, 111(2), 381-389. https://doi.org/10.1006/jcph.1994.1071

Troshichev, O. A., Andrezen, V. G., Vennerstrøm, S., \& Friis-Christensen, E. (1988). Magnetic activity in the polar cap-A new index. Planetary and Space Science, 36(11), 1095-1102. https://doi.org/10.1016/0032-0633(88)90063-3

Wilder, F. D., Clauer, C. R., Baker, J. B. H., Cousins, E. P., \& Hairston, M. R. (2011). The nonlinear response of the polar cap potential under southward IMF: A statistical view. Journal of Geophysical Research, 116, A12229. https://doi.org/10.1029/2011JA016924

Wilder, F. D., Eriksson, S., \& Wiltberger, M. (2015). The role of magnetic flux tube deformation and magnetosheath plasma beta in the saturation of the Region 1 field-aligned current system. Journal of Geophysical Research: Space Physics, 120, $2036-2051$. https://doi.org/10.1002/2014JA020533

Winglee, R. M., Chua, D., Brittnacher, M., Parks, G. K., \& Lu, G. (2002). Global impact of ionospheric outflows on the dynamics of the magnetosphere and cross-polar cap potential. Journal of Geophysical Research, 107(A9), 1237. https://doi.org/10.1029/2001JA000214 